3. Beck S, Abdulla S, Alderton RP et al. (1996) Evolutionary dynamics of non-coding sequences within the class II region of the human MHC. J Mol Biol 255:1-13

4. Kambhu S, Falldorf P, Lee JS (1990) Endogenous retroviral long terminal repeats within the HLA-DQ locus. Proc Natl Acad Sci USA 87:4927-4931

5. Horton R, Niblett D, Milne S et al. (1998) Large-scale sequence comparisons reveal unusually high levels of variation in the HLA-DQB1 locus in the class II region of the human MHC. J Mol Biol 282:71-97

6. Seidl C, Donner H, Petershofen E et al. (1999) An endogenous retroviral long terminal repeat at the HLA-DQB1 gene locus confers susceptibility to rheumatoid arthritis. Hum Immunol 60:63-69

7. Donner H, Tonjes RR, Van der Auwera B et al. (1999) The presence or absence of a retroviral long terminal repeat influences the genetic risk for type 1 diabetes conferred by human leukocyte antigen DQ haplotypes. Belgian Diabetes Registry. J Clin Endocrinol Metab 84:1404-1408

Corresponding author: Dr. J. Martin, Instituto de Parasitología y Biomedicina "López-Neyra", C/ Ventanilla 11, 18001 Granada, Spain

E-mail: martin@ipb.csic.es

\section{DQ-LTR13 modifies Type 1 diabetes (IDDM) susceptibility on high risk DQ haplotypes: reply to the comments of Pascual et al.}

To the Editor: Although we refer to Pascual's study in our original paper [1], these authors like to reiterate their earlier conclusion that $D Q-L T R 13$ is not associated with "autoimmunity" [2]. However the two studies are completely different and therefore not comparable.

Pascual et al. [3] analysed 145 patients with rheumatoid arthritis (RA), 200 healthy controls and 42 selected homozygous cell lines. In contrast we investigated 284 complete families comprising of both parents and at least one child affected by Type 1 diabetes (IDDM) which enabled us to analyse the segregation of 1136 haplotypes with $D Q-L T R 13, D Q A 1$ and $D Q B 1$ alleles and their transmission to patients [1]. Thus our data quality is completely different in comparison to Pascual's study of unrelated individuals which does not allow the assignment of linkage between $D Q-L T R 13$ and $D Q$ in heterozygous individuals. Furthermore we addressed IDDM in Germany and Belgium, whereas Pascual et al studied RA in Spain. Although Pascual et al announce the importance of family studies in their original contribution, they have not obtained any additional data so far. We can therefore only encourage them to carry out transmission distortion tests (TDT) in trio families because only the complete assignment of parental co-transmission or non-transmission can answer the question of an independent $D Q$-LTR13 association with a complex disease such as RA.

We confirm our results leading to the conclusion that $D Q-L T R 13$ is an "additional and independent genetic risk marker" in IDDM. The combined transmission of DQ-LTR13

DOI 10.1007/s00125-003-1114-8

Received: 23 January 2003

Published online: 3 June 2003

C) Springer-Verlag 2003 with selected $D Q A 1 / D Q B 1$ haplotypes and the calculation of the relative risk (RR) clearly indicated that the risk mediated by the $D Q-L T R 13$ is additional to those conferred by $D Q 2$ and $D Q 8$, respectively. The term "independent" referred to the linkage disequilibrium (LD) between the previously reported $D Q-L T R 3$ [4] and DQ-LTR13. This LD was so distinct that both could be considered as independent genetic risk markers.

We differentiated between the transmission of $D Q 8 / L T R 13^{+}$ vs. DQ8/LTR13- and DQ2/LTR13+ vs. DQ2/LTR13- haplotypes in a transmission distortion test and obtained a significant preferential transmission of $D Q 8 / L T R 13^{+}$compared with $D Q 8 / L T R 13^{-}$. $D Q 2$ in contrast was significantly more often transmitted without $D Q$-LTR 13 [1]. This transmission pattern obviously depends on these two haplotypes as we could show a distinct pattern for all not- $D Q 2 /$ not- $D Q 8$ haplotypes that differs for the presence of $D Q-L T R 13$.

Pascual et al. find $D Q$-LTR13 positivity "always" associated with $D Q B 1 * 0302$, *0303 and 0402 alleles. This is in contrast to our results of some DQB $1 * 0302 / L T R 13$-negative as well as DQB1*0201/LTR13-positive alleles. The allele DQB $1 * 0302$ is more often associated with presence of $D Q$ LTR13 than with its absence, but DQ-LTR13 is not confined to the three $D Q B 1$ alleles mentioned above, but also found in $D Q B 1 * 0201(5 \%), D Q B 1 * 0301(7 \%), D Q B 1 * 0501(7 \%)$, $D Q B 1 * 0502(8 \%), D Q B 1 * 0602(4 \%)$ and $D Q B 1 * 0603(5 \%)$ [6]. The discrepancy of the two reports is therefore most probably due to the smaller sample of haplotypes studied in the Spanish data set.

The main interest of our study was to characterize $D Q$ LTR13 as a possible genetic risk marker to IDDM. Previous reports suggesting that " $D Q-L T R 3$ might have an additive effect in the contribution of HLA class II alleles to predisposition to rheumatoid arthritis (RA) and IDDM" [5, 6] represent an earlier state of knowledge and have to be seen in the light of our current data. As clearly pointed out in our publication, the effect of $D Q-L T R 13$ is independent from $D Q-L T R 3$. The massive deletion in the 5'-flanking region of the $D Q B 1$ gene and its association with the absence of $D Q-L T R 3$ in certain $D Q B 1$ alleles described by Pascual et al. has therefore no influence on the results obtained for $D Q-L T R 13$ as it is located outside this deletion.

Due to the complexity of the two diseases and the different populations studied the two reports do not necessarily contradict each other. Our earlier work of a $D Q-L T R 3$ association in German patients with rheumatoid arthritis [5] is currently ex- 
tended to the $D Q-L T R 13$ integration, where we also find a contribution of $D Q$-LTR13 to RA susceptibility in $D Q 8$-positive individuals. Furthermore we recently published that patients with Addison's disease-another HLA DQ8-associated autoimmune disease of the adrenals which may occur in combination with Type 1 diabetes as part of the autoimmune pluriglandular syndrome Type 2 - have more often the DQ8-DQ-LTR 13 combination in contrast to the $D Q-L T R 3$ insertion. Although both $D Q$-LTR 3 and $D Q$-LTR 13 are linked to $D Q 8, D Q-L T R 13$ enhances the risk for Addison's disease [7].

Whether DQ-LTR13 has a functional significance or not is currently under investigation. Preliminary data indicate that $D Q-L T R 13$ harbours regulatory capacity and shows functional activity of an upstream element as revealed by analyses of nuclear initiation of mRNA transcription and steady state cytoplasmic mRNA levels. The further elucidation of this retroviral insertion in the most important IDDM susceptibility haplotype is an important issue.

K. Krach, M. A. Pani, C. Seidl, J. Van Autreve,

B. J. Van der Auwera, F. K. Gorus, R. R. Tönjes,

K. Badenhoop

Center of Internal Medicine, Medical Department I, Division of Endocrinology, University Hospital, Frankfurt/Main, Germany

\section{References}

1. Bieda K, Pani MA, Van der Auwera B (2002) A retroviral long terminal repeat adjacent to the HLA DQB1 gene (DQLTR13) modifies Type I diabetes susceptibility on high risk DQ haplotypes. Diabetologia 45:443-447

\section{Observations}

\section{Compensatory hypochloraemic alkalosis in diabetic ketoacidosis}

Keywords Diabetic ketoacidosis, acid-base imbalance, hypochloraemia

Diabetic ketoacidosis (DKA) is a life-threatening complication of diabetes mellitus. Metabolic acidosis caused by ketoacids is an essential component of DKA and can have detrimental ef-

DOI 10.1007/s00125-003-1119-3

Received: 30 October 2002 / Revised: 21 February 2003

Published online: 11 June 2003

C) Springer-Verlag 2003

Abbreviations: DKA, Diabetic ketoacidosis; [HCO3-], concentration of bicarbonate in arterial plasma; $\left[\mathrm{Na}^{+}\right]$, concentration of sodium in arterial plasma; $\left[\mathrm{Cl}^{-}\right]$, concentration of chloride in arterial plasma; $\left[\mathrm{Na}^{+}\right] /\left[\mathrm{Cl}^{-}\right]$ratio, sodium/chloride ratio in arterial plasma; $\left[\mathrm{XA}^{-}\right]$, concentration of unmeasured anions in arterial plasma; $\left[\mathrm{A}^{-}\right]$, sum of negative charged albumin and phosphate in arterial plasma; $\left[\mathrm{K}^{+}\right]$, concentration of potassium in arterial plasma
2. Pascual M, Martin J, Nieto A et al. (2001) Distribution of HERV-LTR elements in the $5^{\prime}$-flanking region of HLADQB1 and association with autoimmunity. Immunogenetics 53:114-118

3. Donner H, Tonjes RR, Bontrop RE et al. (1999) Intronic sequence motifs of HLA-DQB1 are shared between humans, apes and Old World monkeys, but a retroviral LTR element (DQLTR3) is human specific. Tissue Antigens 53:551-558

4. Donner H, Tonjes RR, Van der Auwera B et al. (1999) The presence or absence of a retroviral long terminal repeat influences the genetic risk for type 1 diabetes conferred by human leukocyte antigen DQ haplotypes. J Clin Endocrinol Metab 84:1404-1408

5. Seidl C, Donner H, Petershofen E et al. (1999) An endogenous retroviral long terminal repeat at the HLA-DQB1 gene locus confers susceptibility to rheumatoid arthritis. Hum Immunol 60:63-68

6. Donner H, Tönjes RR, Bontrop RE, Kurth R, Usadel KH, Badenhoop K (2000) MHC diversity in Caucasians, investigated using highly heterogeneous noncoding sequence motifs at the DQB1 locus including a retroviral long terminal repeat element, and its comparison to nonhuman primate homologues. Immunogenetics 51:898-904

7. Pani MA, Seidl C, Bieda K et al. (2002) Preliminary evidence that an endogenous retroviral long terminal repeat (LTR13) at the HLA-DQB1 gene locus confers susceptibility to Addison's disease. Clin Endocrinol (Oxf) 56:773-777

Corresponding author: Dr. K. Badenhoop, Center of Internal Medicine, Medical Department I, Division of Endocrinology, University Hospital, Theodor-Stern-Kai 7, 60590 Frankfurt/ Main, Germany

E-mail: badenhoop@em.uni-frankfurt.de

fects on cardiac, respiratory and metabolic function [1]. The only known compensatory response to metabolic acidosis in DKA is hyperventilation with consecutive respiratory alkalosis [1].

The effect of chloride on acid-base state has been known for many years. Hyperchloraemia and hypochloraemia cause metabolic acidosis and metabolic alkalosis, respectively [2, 3]. Recent research indicates that changes in chloride play an important role in the compensation of lactic acidosis [4]. Although chloride concentrations are frequently decreased in DKA, it is not known, whether these changes play a role in the acid-base state in this entity. The aim of this study was to investigate the effect of hypochloraemic alkalosis on acid-base state of patients with DKA.

A total of 21 patients with DKA (11 women, 10 men, $44 \pm 16$ years) admitted to the emergency department of a primary care hospital were studied. Fluid, insulin or bicarbonate had not been administered before the investigation. Of these patients, four had new onset diabetes and 17 patients had known insulin-dependent diabetes. DKA was triggered by inadequate insulin dose in three patients, by infection in nine patients and by unknown cause in nine patients. Arterial blood samples were collected from an indwelling arterial catheter and laboratory parameters were measured immediately after drawing. The $\mathrm{pH}$ was $\leq 7.3$ or serum bicarbonate $\leq 15 \mathrm{mmol} / \mathrm{l}$ and ketonuria and glucosuria were present in all patients. The ten healthy control subjects consisted of five women and five men with a mean age of $39 \pm 12$ years.

Acid-base state was assessed using a physical-chemical analysis, which is based on the electroneutrality of plasma. 